\title{
El Endecasílabo en la Poesía Castellana.
}

La reciente conmemoración del cuarto centenario de la muerte de Garcilaso de la Vega ha vuelto a poner de actualidad el problema de la introducción de metros y formas poéticas en España; entre los cuales descuella, por su difusión y aplicaciones posteriores, el versō italiano de once sílabas. Boscán y Garcilaso fueron, en efecto, los gestores del movimiento de renovación literaria que, a principios del siglo XVI, bebió en Italia y aclimató en España el uso de formas de expresión que iban a ser desde entonces casi consubstanciales a la poesía castellana. Endecasílabos, solos o mezclados con heptasílabos, en el metro; sonetos, tercetos, "octava rima", canciones, en la forma, con ellos culminó un proceso de renovación no sólo exterior sino muy íntima en la literatura de Castilla.

Hablar de culminación significa que este movimiento literario había tenido antecedentes. En realidad, aunque la obra de Boscán y Garcilaso fue decisiva en la implantación de nuevas formas, no fue el primer esfuerzo que en el mismo sentido se desarrollara en la península. La primacía de Boscán y Garcilaso fue discutida aún por sus contemporáneos y ha seguido dando ocasión a importantes estudios li- 


\section{$-386-$}

terarios, entre los que sobresalen los valiosos informes de don Marcelino Menéndez y Pelayo en diversos pasajes de sus prólogos a la Antología de poetas líricos castellanos.

Dejando de lado las observaciones de carácter exclusivamente métrico, vamos a ordenar y resumir ciertos aspec. tos de la historia del metro endecasílabo en Castilla antes de Boscán y Garcilaso, agregando algunos datos que puedan ser de utilidad para los estudiantes universitarios.

\section{Primeras manifestaciones.}

Es difícil señalar con exactitud la más antigua aparición del verso de once silabas en la poesía castellana. La pérdida indudable de cantares de los primeros tiempos, $y$ las -irregularidades métricas de que, por impericia técnica, defectos de la lengua o licencias del canto juglaresco, estaban cuajados los poemas, hacen que no se pueda precisar el número exacto de sus sílabas. 'Además, sin necesidad de un mayor estudio es fácil comprender que en la mayor parte de los casos la primitiva medida métrica ha de haber llegado hasta nosotros modificada o deformada por descuidos de la tradición oral o los copistas.

De todos modos, lo que parece cosa averiguada es que la medida de once sílabas tiene sus antecedentes innegables en la literatura clásica. Los críticos han analizado la manera cómo se derivan, de los originarios metros greco-latinos, nuevas formas poéticas que no sólo hacen invariable el número de sílabas (antes modificado por la compensación de largas y de breves) sino, olvidando la cuantidad prosódica, fijan su nota capital en la determinación de los acentos rítmicos. En lo que se refiere a los versos de once sílabas, esa derivación da por resultado en las lenguas romances tres versos de índole análoga, pero con particularidades diferentes: el decasílabo épico francés, el endecasílabo lírico provenzal y el endecasílabo italiano. 
Del decasílabo épico francés (considerado de diez sílabas por la manera especial de contar las sílabas en Francia), prácticamente no hay manifestaciones en la epopeya castellana. Menéndez y Pelayo cita como ejemplo discutible un verso del Poema de Mío Cid; precisamente el primero en el códice de Per Abbat. Acomodando a la medida francesa la palabra "oios", y convirtiendo en muda la "e" final de "fuertemientre" (como era corriente hacer en los cantares de gesta y aún mucho después en los romances), podría decirse que tenemos un decasílabo épico "a minori", es decir con acento y pausa en cuarta sílaba:

De los sos oios / tan fuertemientr(e) lorando....

En cambio, del endecasílabo lírico provenzal encuentra copiosas y muy señaladas derivaciones, en las dos formas cngendradas casi simultáneamente en la península: el endecasílabo catalán y el endecasílabo galaico-portugués. Ambos campos se hallan, en rigor, fuera de la intención de estos apuntes, que sólo se refieren a la historia del metro de once sílabas en la literatura estrictamente "castellana". Pero no se puede dejaredeurecordar los nombres de algunos trovadores catalanes, como Guillem de Bergadan o Serverí de Gerona, cuyos endecasílabos en lengua provenzal son los determinantes de la cesura (y ya no sólo pausa fuerte) en cuarta sílaba, que caracterizó el uso de esta madida en Cataluña.

Menos desarrollo, pero mayor relación con la literatura castellana, tuvo el endecasílabo gallego; o, por mejor decir, galaico-portugués, ya que hasta el siglo XV no podemos hacer con fundamento una separación esencial entre ambas lenguas. Sabido es que la primera poesía lírica en Castilla se escribió en galaico-portugués, que fue su instrumento casi obligado de expresión. Y así es en las "Cantigas" del monarca castellano Alfonso el Sabio (1252-1284) donde encon- 
tramos, por primera vez en la zona central de la península, versos regulares de once sílabas:

De mi gran fermosura una doncella...

En los Cancioneros de Ajuda, del Vaticano y ColocciBrancuti encontramos muchos ejemplos semejantes. Los poetas gallegos, herederos como ninguno del sentido lírico y la riqueza métrica de los trovadores occitánicos, no sólo utilizaron con frecuencia el endecasílabo provenzal sino adoptaron una forma muy característica: la llamada, precisamente por esta razón, de "gaita gallega" o, como quería Milá y Fontanals, "anapéstica". Lo determinante del endecasílabo anapéstico es el acento obligatorio en las sílabas cuarta : séptima; y el frecuente, pero voluntario, en la primera. Esto le dá un carácter especial, muy propio para el canto y de indudable brío rítmico. Cuando. lleva también acento en la primera, podemos decir que tenemos un verso en cuatro partes: tres anapestos (pie compuesto de dos breves y una larsa) y una sílaba inicial; $₫$. ej.

\section{Quel mayor poss'a o mays encoberto...}

(Canc. Vat.-N." 115 )

Este carácter hace que el endecasílabo anapéstico sea fácil de resolverse en otros metros. 'Así, por ejemplo, en este caso, en que se convierte en un verso de docé por añadidura de una sílaba inicial:
A Santa María fiz hir meu amigo...
- (C. Vat.-N.0 722)

O bien el caso contrario: pérdida de una sílaba, aún por simple contracción al pronunciarla, volviéndose un verso de diez sílabas: 


\section{$-389-$}

Mays entendo de vos una rem...

(C. Vat.-N.० 464)

En todos estos ejemplos se trata de poesías en idioma galaico-portugués. Para hallar endecasílabos en castellano en el siglo XIII habría que acudir al método indirecto de buscar muestras ocasionales, producidas por la irregularidad en el cultivo de otros metros. Así, en el Pocma de Santa María Egipciaca lecmos versos como éste:

Todos aquellos que a Dios amarán...,

que no es, en rigor, sino una descuidada traducción del endecasílabo francés característico de las "Vidas de Santos".

El endecasílabo en el sigilo xiv.

¿Cuál es, cntonces, la más antigua manifestación del endecasílabo en castellano?

La primera ocasión en que do encōntramos en una forma precisa y deliberada es en el célebre Iibro de Patronio o "Conde Lucanor", del Infante Don Juan Manuel; termina(lo, según propia declaración, el año de $\mathrm{I} 338$. "El Conde Lucanor" es una obra en prosa (brillante y valiosísima colección de relatos que inicia, con el "Decamerón", la moderna novelística europea), en que su autor cuida de acentuar la enseñanza, el "castigo" que se debe derivar de cada cuento. Estas moralejas son pareados en verso; y en varias ocasiones están compuestas en metro endecasílabo, con terminaciones agudas, graves y aún esdrújulas:

Non te espantes por cosa sin razón, Mas defiéndete bien como varón. 
Non aventures mucho tu riqueça Por consejo del ome que ha pobreça.

Non castigues al moço maltrayéndole, Mas dile como vayas aplaziéndole.

También en el otro gran escritor del siglo XIV, Juan Ruiz, Arcipreste de Hita, la figura sin duda más representativa de la literatura española en la Edad Melia, podemos hallar algún ejemplo. El 'Arcipreste pertenece al mester de clerecía; pero en las canciones que intercala en el "Libro ce Buen Amor" rompe los moldes formales del mester para alegrar, con notas ágiles y a la par vigorosas de expresión, el paso un tanto lento del alejandrino monorrimo. E1 mismo, dejando de lado sus repetidas declaraciones de ser "escolar rudo", llega a decir, en la Introducción, que compuso su obra: "otrosí a dar algunos lección c muestra de metrificar, e rrimar e de trobar; ca trobas e notas e rrimas e ditados e versos, que fiz complidamente segund que esta cioncie requiere""

Cejador ha señalado en el "Libro de Buen Amor", además de los versos de I4 sílabas del tetrástrofo clásico, otros de 4 , de 5 , de 6 , de 7 , de 8 y de I6. De i i sílabas en 'rigor' no hay ninguno. Pero si juntamos (como hicieron entre otros Puyol y Menéndez y Pelayo) los versos de 4 y 7 sílabas de una de las "Cantigas en Loor de la Virgen", tendremos una manifestación cumplida y a la verdad muy elegante del endecasilabo:

Quiero seguir - a tí, flor de las flores, Siempre decir - cantar de tus loores...

(Coplas 1678 y sigs.)

Después de este ejemplo ocasional no volvemos a encontrar endecasílabos en Castilla hasta los poetas de la impor- 
tante época de transición del siglo XIV al siglo XV, cuyas composiciones se hallan recogidas en el Cancionero de Baena. Sabido es que en este Cancionero, de tanta trascendencia para la historia literaria, se agrtpan producciones de dos tendencias definidas: una, la continuación de los trovađores galaico-portugneses, y otra, la imitación de la poesía alegórica italiana. En la printera escuela no hay en realidad cultivo del metro endecasílabo. Tos ejemplos que se descubren, fuera de ser muy esporádicos, representan, más que andecasílabos anapésticos, alteraciones en la medida afín y bastante común en esa escuela, de los versos llannados de "arte mayor", de doce sílabas. Perdida la sílaba inicial, tenemos algunos casos en apariencia de "gaita gallega"; cono en estos versos de Alfonso Alvarez de Villasandino, representante principal de la escuela:

Sepan que es árbol de grand maravilla,

Tío del alto león de Castilla,

E de la lyna Rreal de Levante..

$$
\text { (Canc. de B. -N. }{ }^{\circ} \text { ); }
$$

o estos otros del Comendaclor Ferrán Sánchez Talavera:

¿Qué se fisieron los Emperadores,...

¿Padres e fijos, hermanos, parientes...

(C. de B.-N. $\left.{ }^{\circ} 53^{\circ}\right)$

En tho y otro caso, se trataría solamente del endecasílabo gallego. Ia introducción del endecasilabo italiano-verso más libre y de mucho mayor aplicación, porque reemplaza la monótona y acusada cesura en cuarta sílaba por una detención poco ostensible-la iba a realizar la segunda escuela representada en el Cancionero de Baena. Aunque 


\section{- $392-$}

iniciada todavía en el siglo XIV, su obra iba a tener por campo principal los primeros años del siglo siguiente.

El endecasílabo en el siglo XV.

Es, efectivamente, en la primera mitad del siglo XV cuando comienza a afluir con regularidad a tierras de Tispaña la armoniosa corriente del endecasílabo italiano. Ia importancia que tuvo descle su aparición y el interés polémico que despertara-si no tanto por sí, como instrumento del arte alegórico de Italia-, revelan que lo que se trataba era, no un simple asunto literario, sino un movimiento más complejo. Lo que empezaba a ponerse en discusión era nadı menos que el cambio de gusto, la transformación del carácter cultural por influencia de los autores del primer Renacimiento, con la consiguiente desviación del meridiano intelectual de Francia a Italia.

Hasta entonces, como es sabiclo, la influencia extranjera predominante en la literatura castellana había siclo la francesa: tendencia a la regularidad métrica e impronta de la influencia novelesca $\mathrm{y}_{\text {in }}$ sentimental en los cantares; influencia de la poesía provenzal y posición elevada en Castilla de los trovadores occitánicos; imitación, y aún traducción literal en ciertos casos, de las Vidas de Santos; difusión de las versiones francesas, tanto de los poemas pseudo-clísicos como de la copiosa literatura eclesiástica, especialmente las leyendas marianas; reflejo un tanto episódico pero indiscutible de los "fabliaux" franceses y las alegorías morales, como la batalla de Don Carnal y Doña Cuaresma en el "ILibro del Buen Amor", del Arcipreste de Hita; etc. Pero en el siglo XV la situación varía: el país que se observa, aquel cloncle se bebe la cultura y al que se considera heredero de una literatura-que precisamente entonces no sólo se restaura sino se coloca en un plano de superioridad y ejemplariclarl inobjetables-es Italia. Debilitado el cuerpo, un tiempo mag- 
nífico por su organización interior, de la Edad Media, a Italia se dirigen las miradas, para encontrar, junto con la resurrección de las obras maestras de la literatura de Grecia y Roma, una nueva perspectiva intelectual y un sentido más amplio y más armonioso de la vida.

La labor cultural que se desarrolla entonces en Italia, y que antecede en calidad y en previsión histórica a la de los demás países de Occidente, había de atraer indudablemente la atención de los intelectuales de las otras naciones, especialmente las cercanas.

Concurrían a hacer mayor esa atención varios factores de importancia. En primer lugar, la muy eficaz forma de estímulo que representaba el desarrollo del idioma toscano. Las obras de Dante, Petrarca y Bocaccio tenían, además de su valor intrínseco y de su capacidad de resonancia-en una época en que los problemas europeos eran en esencia tan comunes-, el especialísimo interés de estar compuestas en una lengua, afín a otros idiomas en su desenvolvimiento, pero que alcanzaba antes que ellos y como un ejemplo para ellos, la más alta dignidadeliteraria. Por primera vez una lengua romance llegaba no sólo a equipararse al latín por su riqueza, su fuerza expresiva, su facilidad para adaptarse a todas las manifestaciones del espíritu, su arquitectura lógica, su opulenta y armoniosa elegancia, sino aún podía sobrepasarla en la flexibilidad y el libre juego, que un genio más sintético y unas leyes bastante más estrictas no habían permitido a la rotunda lengua madre de que el toscano procedía.

A estos factores de carácter general se unían, en el caso de España, muy especiales circunstancias históricas. Las razones políticas empezaban a mover, y cada vez en mayor número y con más evidente intensidad, a los ejércitos españoles en Italia. Especialmente los Reyes de Aragón, que con la derrota de Muret (en г2r3) habían cancelado sus ambiciones en el campo occitánico, dirigían ahora su atención y 
sus esfuerzos hacia Italia; hasta lograr, después de varias vicisitudes (entre ellas el fracaso de Gaeta), (jue uno de sus monarcas, Alionso $V$, entrara en completal posesión 170 sólo de tierras insulares, como Sicilia y Cerdeña, sino del próspero e importante Reino de Nápoles (1443).

Por último, hay que considerar una nueva y ya muy vital forma de enlace: la razón econónica. A las refaciones culturates, a la influencia de las Lniversidades, a las peregrinaciones religiosas, a la directa y esforzada actuacion de las expediciones militares, venia a unirse asi, para completar la intercomunicación de ambas penínsulas, el elemento consercial, de importancia a cada momento más creciente. Conocidos son el desarrollo en este orden y la organización de instituciones comerciales en las ciudades ifalianas. Ese desarrollo se manifestó en el exterior, no sólo en el cirvío r. recepción de mercadería o documentos, sino en la instalación, cada vez en mayor número, de conerciantes italianos, en especial en los países vecinos. Estos comerciantes, ya refinados y entiquecidos, o más comúmmente la generación posterior que aprovechaba la comodidad económica que había ganado sn familia, contribryeron en gran nodo a difrundir el moviniento cultural cle su país de origen, dándose a la lectura, a la versión o a la imitación, más o menos lograda, de los autores latinos e italianos.

Fute precisamente en esta forma cómo se introdujeron en España, de manera concreta, los temas y los metros de la literatura italiana del primer Renacimiento. El introductor fue un italiano, Micer Francisco Inperial, hijo de tutr mercader genovés de joyas, avecindado en Sevilla descle mediados del siglo XIV. Imperial tenia un espiritu poético innegable ("no lo Ilamaría deciclor o trovador, mas poeta",escribia el Marqués de Santillana) y tun abundante y muy familiar conocimiento de los autores de mayor influcncia entonces en Italia. (EI mismo cita a Homero, Virgilio, Horacio, Ovidio, Ltucano, Boecio, Dante). Tenía además hasta 
una especial facilidad para el manejo de varias lenguas, cono el frances, el inglés y el árabe; fuera, desde luego, del castellano y el italiano, que clominaba perfectanente.

Pero, a pesar de la variedad de sus lecturas, Imperial sentía, sobre todo, una viva, apasionada y casi monopolizadora simpatía por Dante. La influencia de Dante está en sus obras líricas, politicas, amorosas, morales; está en su introducción de la alegoria, tan caracteristica de Dante y qus tanta extensión iba a alcanzar en la literatura castellana; $y$ está, por último, entre otros muchos aspectos, en el que nos interesa más en este instante: la introducción deliberada del mdecasílabo italiano. Micer Francisco Imperial inicia una campaña concreta, cleliberacla, intencional, para familiarizarlo en las tierras ibéricas. Desde luego que en th poeta de esta época, que es esencialmente de transición entre dos espíritus, no es dificil hallar ciertas imprecisiones de factura e irregulariclades métricas, algutnas de las cuales pueden no ser atributibles a él sino-cono estima Menéndez y Pelayo-a la negligencia con que transcribió sus versos el copista del Cancionero de Baena, todaría no acostumbrado ai nuevo metro. Entre otros descuidos de detalle, podemos observar en las poesias cle Imperial atin ef el "Dezy delas"siete virtudes", que es su obra maestra--alguna mezcla poco experta y no grata al oido, de enclecasílabos de cliversos órdenes, yámbicos, sálicos, anapésticos; conno:

Cerca la hora que el planeta enclara.

Ena en la vista benigno e sïave.

Barba e cabello alvo syn mesura; etc

Pero esto no obsta para reconocer la señaladísima importancia de Imperial como introductor del endecasílabo italiano en España; empresa en la que podría decirse que logró 
lo que pedía con tanta nobleza en su emocionada invocación a Dante:

E faz mi lengua tanto meritoria

Que una scentella sol de la tu gloria

Pueda mostrar al pueblo aquí presente.

La obra poética y de cultura de Imperial se había desarrollado en Sevilla. Allí le rodeó y lo continuó una copiosa promoción de escritores, como Páez de Ribera y los Medina; y de allí la llevó a Castilla el sevillano Ferrán Manucl de Lando, activo propagandista y sostenedor de una tremenda y muy agria polémica con los representantes de la tradición trovadoresca. Pero si el nuevo espíritu y la adaptación de la alegoría dantesca consiguieron el triunfo y se difundieron ampliamente, no sucedió lo mismo con el endecasílabo, que se vió pretericlo por el verso de arte mayor o de cloce sílabas, que llegó a ser el más común y hasta cierto punto el distintivo de la época. Puede decirse que es sólo en los momentos de descuido de este metro, que asoman versos de once sílabas en los compañeros de Imperial. Así, en Ruy Páez de Ribera prge Puccinelli Converso"

Dizen los sabios: "Fortuna es mudable"...

(C. de B.-N. ${ }^{\circ} 289$ );

en Gonzalo Martínez de Medina:

Tú que te vees en alta coluna...

(C. de B.-N. ${ }^{\circ} 339$ );

o en el gracioso poema que se ha llamado "de los castillos en el aire", de Pero González de Uceda:

Cuando me cato con grand ligereza

Véome en Flandes merchante tornado. 
Lindo, fidalgo, garrido et donoso.

El único que recogió la labor cle Imperial respecto al endecasílabo y le dió un nuevo y muy valioso impulso, fue el Frócer y altísima figura poética del siglo XV: Don Iñigo López de Mendoza, Marqués de Santillana. El Marqués de Santillana vuelve a adoptar el nuevo metro y a componerlo en una de sus formas más características, en sus 42 sonetos "fechos al itálico modo"; i 7 dirigiclos junto con la "Comeclieta de Ponza" a la Condesa de Módica, Doña Violante de Prades, y 25 escritos posteriormente. A pesar de sus conocimientos literarios, el modo no es del todo itálico, porque tiene especiales variaciones en las rimas de los cuartetos y hasta ciertas imperfecciones en la misma medida; mas la lozanía y la gracia del Marqués le hacen lograr algunos aciertos innegables como en el muy hermoso soneto que comienza:

¡Oh dulce esguarde, vida e honor mía, Segunda Elena, templo de beldat. So cuya mano, mando e señoría

Es el arbitrio mio e voluntat!

También se encuentran endecasílabos, pero de un carácter bien distinto, en su contemporáneo e íntimo amigo Juan de Mena. Son, más bien, disonancias, que, por la frecuencia con que se hallan y dacla la maestría técnica de su autor, se han considerado como alteraciones intencionales en los versos de doce sílabas tan característicos de Mena. Sería así un caso de lo que Nebrija llamaría verso "cacómetro cataláctico", que no dura sino una línea y se enmienda en seguida, como en la siguiente estrofa del "Labyrintho", que comienza con un verso de once sílabas:

¡Oh, virtüosa, magnífica guerra!..., 


\section{$-398-$}

y sigue luego con dodecasílabos regulares:

¡En tí las querellas volverse debriatn..., etc.

Disonancias sennejantes, aunque de un carricter difícil de precisar, se puede observar en varios poetas del siglo XV; a veces en estancias completas, como la frimeta del "Dezyr" a la muerte de D. Diego Hurtado de Mendoza, de Fermín Pérez de Guzmán (ya señalacla por Menéndez Pelayo), que comienza :

Onbre que vienes aquí de presente.

Tú que me vistes ayer Almirante,...

(C. de B.-N. $57 \mathrm{r}$ );

o en Carvajales:

Supo su mal e su gloria perdida...

(Serranilla);

en Diego del Castillo:

Era "llegada la noche oceanas so"

(Vision sobte la muerte del rey Don Alfonso);

en Gómez Manrique:

Nueve centenas e una después...

(Defunción del noble caballero Garci-Lasso de la Vega);

en el meritísimo Juan del Enzina:

Tú que llevabas a Belerofonte...

(Tragedia trovada a la colorosa muerte del príncipe Don Juan);

en el Condestable Don Pedro de Portugal: 
Vos sulbudgades, faziendo vos viles...

(Coplas de contempto del mundo);

o en el también portugués, pero autor de versos castellanos, Don Juan Manuel:

Más donde ama que no donde anima...

(A la muerte del príncipe D. Alfonso).

Por fin, en dos obras anónimas del mismo siglo XV en.. contramos también otros endecasílabos esporádicos. En la "Revelación de un ermitaño", que dá la fecha de I420, dice el cuerpo al alma:

Tú mi sennora, yo tu servidor.

Y en la "Danza de la muerte", obra traducida del francés probablemente a mediados del siglo $\mathrm{XV}$, leemos cuando habla el Padre Santo:

Ay de mî, triste, qué cosa tan fuerte...

De índole distinta, pero que a la postre resulta coincidente, es el movimiento literario del siglo XV en las regiones de Valencia y Cataluña. A pesar de la cercanía geográfica y de las relaciones culturales antudadas de antiguo con Italia, no se produce verdaderamente un influencia del endecasílabo toscano. Y ello no por un rechazo de este metro, sino precisamente porque, a diferencia de Castilla, el verso que más había arraigado en esta zona era otra clase de endecasílabo: el de tradición provenzal, con acento y pausa en cuarta sílaba. Así lo vemos en sus principales poetas, descle el vigoroso, conciso y trascendente valenciano Ausías March, hasta algunos autores menos graves como Mosén Jordi de Sant Jordi y Roig de Corella. Generalmente-y siguiendo en esto el carácter de 
la lengua-, el acento en cuarta se proiluce con una terminación aguda.

Aunque la literatura en lengrua no castellana desborda la intención de estos apuntes, no se puterle dejaj de citat algunas lineas de Ausias Mauch como ejemplo de su inanejo peculiár de la medida enrlecasilaba:

La gran dolor, / que lengua no pot dir,

Del quis veu mort / e no sab hon irá,

No sab son Deu / si per a s'il rolrá

O si n'infern / lo volrá sebollir...

Sólo Roig de Corella compensa en algo el acento fijo en cuarta sílaba, dindole al verso otras acentuaciones que se ha creído no deliberadas "y por lo mismo más significativas":

Ab plor tan gran que nostres pits abcura,

E greu dolor que 1 nostre cor esquinça...

Lo vostre cor partit aj fort escarpre...

\section{I-A IN Novación DE Boscín Y Garciraso.}

De esta manera se hallaba ya el camino completamente preparado. En Castilla y Cataluña se había creado, por razones distintas $\mathrm{y}$ con diferentes aspectos en su desarrollo, un común novimiento de renovación de las formas $y$ del gusto, que iba a llegar a su culminación a principios del siglo XVI. Como escribe Menéndez y Pelayo, se había ido "preparando la hora solemne en que los discípulos de Micer Francisco Imperial, de Juan de Mena y del Marqués de Santillana, habían de encontrarse con los de Jordi y Ausias March en el puerto de Barcelona, y reconociendo la fuente comín de sus inspiraciones, habian de sellas el pacto de alianza por manos de los Dioscuros de la lírica ítalo-hispana, Boscán y Garcilaso". 
Sin embargo, esa transformación de raíces tan profunLas y tan preparada a través de un siglo, no vino a deterninarse en realidacl sino por tul motivo ocasional. Entre los que cotaboraban, en diversas esferas y con diferente intensidad, a las relaciones cntre las dos penínsulas, se contaban, junto it soldados, religiosos, hombres de estudio, comercianes, los diplomáticos--entonces especialmente cultos-que se cruzaban y se intercomunicaban en sus viajes. Uno de esos diplomáticos ftue Andrea Navagero, Embajaclor de la Señoría de Venecia ante España, de 1524 a 1528 , y uno de los más completos $y$ sobresalientes humanistas italianos de entonces. Navagero no se dedicó sólo a las labores de su cargo cliplomático-que las citcunstancias de la época no permitieron hacer más eficientes-, sino desarrolló tuna actividad intelectual de primer orden. Viajó, observó, analizó; escribió cartas importantes sobre España; se rodeó cle amigos literatos; y fue precisamente a sus inslancias que el catalán Juan Boscán Almugaver emprendió la tarea de trasladar al castellano los metros y formas rítnicas de Italia.

El propio Boscíln ha narrado este episodio, de tan honda importancia para la listoria litemeria, enda conocicla carta a la Duquesa do Sorta? que Obras completas: "...Porqque estando un día en Granada con el Navagero (al qual, por haher sido varón tan celebrado en nucstros días, he querido aquí nombralle a vuestra Señoria) tratando con él en cosas de ingenio y de letras, y especialmente en las variedades de muchas lenguas, me dixo por qué no probaba en lengua Castellana Sonetos y otras artes de trovar usadas por los butenos autores de Italia; y no solamente me lo dixo así livianamente, mas aun me rogó que lo hiciese. Fartíme pocos días después para mi casa, y con la largueza y solectad del camino disctrriendo por diversas cosas, fuí a dar muchas veces en lo que el Navagero me haJía dicho; y así comencé a tentar este género de verso. Eñ el qual al principio hallé alguna dificultad, por ser muy artifi- 
cioso y tener muchas particularidades difrentes del nuestro. Pero después pareciéndome, guiza con el amor de las cosas propias, que esto comenzaba a sucederme bien, fuí poco a poco metiéndome con calor en ello".

Boscán, por lo demás, aunque en la primera parte de su carta dice del conjunto de st empresa que era "cosa nueva" en España, no desconoce algumos antecedentes en el uso ciel verso endecasílabo, que le sirven precisamente para reforzar st implantación. Así, después de citat a Ausias March observa que "aun volviendo més atrás de los Provenzales, hallaremos todavia el camino hecho deste nuestro verso. Porque los endecasílabos, de los quales tanta fiesta han hecho los latinos, llevan casi la misma arte y son los mismos en quanto la diferencia de las lengutas lo sufre". Y añade: "porepte: acabemos de llegar a la fuente, no han sido dellos tampoco inventores los latinos, sino que los tomaton de los griegos, como han tomaclo otras muchas cosas scñaladas en diversas artes. De manera que este género de trovas, y con la autoridad de sut valor propio y con la reputación de los antiguos y modemos que le lian usado, es cligmono solanıente de ser recibiclo en una lengua tan buena como es la Castellana, mas aun de ser en elia preferido a todos los versos vulgares".

Boscán usó exclusivamente los metros italianos en la segunda y tercera parte de sus obras, pero su versificación es todavía un tanto dura y en ocasiones hasta ingrata. Se le reprocha, sobre todo, las terminaciones agudas, que algunos atribuyen a su nacimiento catalán, y que disuenan con la apacibilidad de voces graves del endecasilabo italiano. Otras veces los defectos, a más de técnicos pueden considerarse de interpretación, como en el uso de sinalefas y de hiatos, y aún discordancias en la acentuación que dan al verso un carácter cortado y no fluido.

Para dar idea de algunas de estas asperezas-que aburndan, por desgracia, aún entre sus prodtcciones más logradas-, podemos señalar los siguientes versos: 
Y el desgusto que del sufrir me alcanza...

En mí presto se acabará el tormento...

Si parto, sólo por irme me voy.

Pero otras veces, desde luego, el resultado induclablemente es muy hermoso:

Las cejas son los arcos que Annor flecha;

Ios rayos te los ojos las sactas

Que sullaga mortal traen muy hecha.

¡Oh, multitud de gracias tan perfetas,

Que su cuenta al contar si justa se echa

Es para enmudecer cien mil poetas!...

(Octava rima)

La labor de Boscán no hubiera podido, sin embargo, alcanzar la diftusión y la aclinatación que pretendia, de haber estado reducicla a sus propios recursos. Por las deficiencias ya indicadas y pos la evidente dalta de vtelo Tirico-que habría de linitar sieñmpesus composiciones, aún en el caso de lograr una pericia técnica que, como hemos visto, le faltaba - su innovación no hubiera pasado de un ensayo exclusivamente personal. Su vida habría siclo posiblenente tan elímera como la de las introducciones italianas de Imperial n los sonetos del Marqués de Santillana que, sin dejar verdadera huella en la historia literaria, podria clecirse en realidad que habian terminado con sus propios autores.

Pero la obra cle Boscán tuvo un refuerzo clecisivo. Fué la adhesión, el entusiasmo, y sobre todo la conperación en la labor, del más dilecto amigo suyo: el poeta Garcilaso de la Vega, una de las voces más puras y más finas de la literatura castellana. Sabicla es la amistad que ligó siempre a estos poetas; anistad iniciada en plena mocedad, al encontrarse 
en la corte del Emperador en I 520, y que sobrevivió a la tumba desde que fue por cuidado de la viuda de Boscán, Doña Ana Girón de Rebolledo, que las obras poéticas de ambos se publicaron por primera vez juntas, en un solo volumen, cuando los dos habían fallecido.

Boscán reconoció siempre ampliamente la importancia del apoyo que había recibido de Garcilaso. En la misma carta a. la Duquesa de Soma en que habla de su implantación de formas y metros italianos, declara que no hubiera obtenido buen éxito en su empeño si Garcilaso "con su juicio, el qual no solamente en mi opinión, mas en la de todo el mundo, ha sido tenido por regla cierta", no lo confirmara en su deman-da. "Y así alabándome mitchas veces este mi propósito-añade-, y acabándomele de aprobar con su exemplo, porque quiso él también llevar este camino, al cabo me hizo ocupar mis ratos ociosos en esto mas particularmente".

En realidad, Garcilaso tenía todas las condiciones que le faltaban a Boscán. De noble cuna, con un largo y brillante abolengo literario (era descendiente del Canciller Pero López de Ayala, de Fernán Pérez de Guzmán y del Marqués de Santillana, entre otros), unía a su nativo refinamiento y a su esmerada educación, el perfeccionamiento literario que representaban su vida en Italia y su contacto con las principales figuras intelectuales de aquel país, especialmente en Bolonia y en Nápoles. De ese contacto obtuvo Garcilaso una absoluta compenetración con los modos y gustos que se trataba de implantar, y aún la ratificación final de un oído, no duro como el de Boscán, sino acostumbrado a percibir la música y las cadencias italianas.

La poesía de Garcilaso tiene, por éso, una dulzura, una pureza y una elegancia extraordinarias. Con él nos hallamos en un mundo que si puede ser débil, convencional e indudablemente poco personal en el fondo, desde el punto de vista de la forma representa uno de los momentos más logrados de la poesía castellana. Es el lenguaje poético preciso para 
Ios exquisitos sentimentalismos italianos: sonoridad, flexibilidad, períodos amplios, frases optlentas en un senticlo y de una sutil ternura en otro, melodía, apacibilidad, fluidez, elegancia. En una palabra, música. "Música ante todo", podía haber siclo, cn efecto, el lema de este poeta aristocrático, con la frase que, siglos después, iba a servir de epígrafe a uno die los poemas de Verlaine.

Garcilaso sigue así una línea melódica que hasta entonces había sido casi desconocicla en la poesía-predominantemente vigorosa y no de ritmo blando-de Castilla. I as consonancias son cuidadas; los períodos redondos; las terminaciones-tanto en el endecasílabo, que es su verso más característico, como en los otros metros-se libran de la tendencia al agudo de Boscán, para utilizar las palabras graves que, fuera de su mayor cercanía al italiano, otorgan a la poesía indudablemente un aliento más amplio y una elegancia más pausada. Tenemos, de tal modo, en Garcilaso endecasílabos maravillosos de frescura:

Corrientes aguas, puras, cristalinas, árboles que os estâis mirando en ellas, verde pradorde fresca sombra lleno, aves que aquí sembráis vuestras querellas...;

(Egloga primera);

endecasílabos de un firme brío lírico:

¡Oh, dulces prendas por mi mal halladas, dulces y alegres cuando Dios quería!...

(Soneto X);

o con la idílica y ponderada melodía de sus descripciones de paisajes:

Movióla el sitio umbroso, el manso viento, el suave olor de aquel florido suelo. 


\section{$-406-$}

Las aves en el fresco apartamiento

vió descansar del trabajoso vuclo.

Secaba entonces el terreno aliento

el sol subido en la mitad del cielo.

En el silencio sólo se escuchaba

un susurro de abejas que sonaba.

(Egloga tercera)

Era la introducción definitiva del endecasílabo italiano. Con la autoridad de Garcilaso y con la fucrza admirable c incontrastable de su ejemplo, los poetas de los tres grandes campos lingüísticos de la península ibérica se encontraron con una forma de expresión de la que ya no podrían prescin-dir por su depuración y su eficacia. De nada iba a servir la oposición de Castillejo. Los propios partidarios de la escuela tradicional-Gregorio Silvestre, Gálvez de Montalvo, Tope de Vega en sus obras de juventud-tuvieron que terminar por hacer versos en la nucva manera. La aclimatación llegó a ser tan perfecta que hasta se sostuvo que el verso de once sílabas no era en realidad italiano sino de ascendencia española. Así lo expresa Juan de la Cueva en su "Exemplar poético"; JyrArgote de Molina, al Preferirse al endecasilabo, afirma que "al cabo de algunos siglos que anduvo desterrado de su naturaleza ha vuelto a España”. El cndecasílabo se convirtió en el metro obligado para los asuntos de mayor seriedad y trascendencia. Juan de Coloma, en su "Décacla de la Pasión de Cristo", declara que escogió el terceto (en versos de once sílabas), "porque es el metro más grave y majestuoso que ticne la lengua y se acomoda admirablemente a argumentos graves". "Son los tercetos para cosas graves", habría de añadir más tarcle Lope de Vega en su "Arte nuevo de hacer comedias". Un paso más, y los particlarios de los antiguos metros adoptarían una actitud, ya no ofensiva sino defensiva. "Después que Garcilaso de la Vega y Juan Boscán-escribía Hernando de Hozes en el 
prólogo de su traducción de los "Triunfos" de Fetrarca, en fecha tan cercana a la innovación cono 1554 -truxeron a nuestra lengua la medida del verso thoscano, han perdido con mucho tatuto crédito todas las cosas hechas o traduzictas en cualquier género de verso de los que antes en España se usaban, que ya casi ninguno las quiere ver, sienclo algunas (como es notorio) de mucho precio".

$\mathrm{Si}$, con la frase de Migutel Attigas, "Ta lengua de Gatcilaso tiene tn sabor a fruta madura", en lo gue se refiere al la parte métrica puede decirse también que, desde entonces, el verso italiano de once sílabas queda definitivamente instatuado en Castilla con todo el sabor, la lozanía, la riqueza intina de la fruta en sazón.

$$
\text { Jima, } 1936 \text {. }
$$
A. Miró Quisada S.

\section{Biblioteca de Letras} "Jorge Puccinelli Converso»

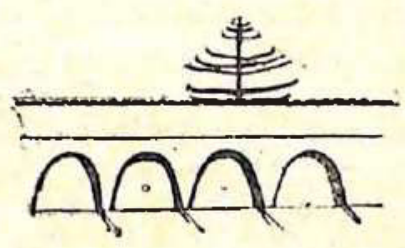

